\title{
Hair Loss Associated with Sertraline: Two Case Reports and Review
}

\author{
Forouzan Elyasi ${ }^{1,2, *}$ \\ ${ }^{1}$ Department of Psychiatry, School of Medicine, Mazandaran University of Medical Sciences, Sari, Iran \\ ${ }^{2}$ Psychiatry and Behavioral Sciences Research Center, Addiction Institute, Mazandaran University of Medical Sciences, Sari, Iran \\ "Corresponding author: Forouzan Elyasi, Psychiatrist, Assistant Professor of Psychiatry, Consultation-Liaison Psychiatry Ward, Imam Khomeini Hospital, Sari, Iran. Tel: \\ +98-9111551097, Fax: +98-1133363754, E-mail: forouzan.el@gmail.com
}

Received 2015 September 03; Revised 2016 April 07; Accepted 2016 September 23.

\begin{abstract}
Introduction: Selective serotonin reuptake inhibitors (SSRIs) are widely advised as antidepressants. Association of SSRIs with hair loss is rare and limited to a few case reports. In this case study, two patients developed diffuse hair loss during sertraline treatment. Case Presentation: Mr. A was a 47-year-old married man with a diagnosis of panic disorder according to the DSM-V criteria and specific phobia (claustrophobia). Approximately 8 weeks after starting sertraline intake, he noticed hair loss. Decreasing the dose of his medication led to the resolution of hair loss within 6 weeks. Ms. M was a woman with major depressive disorder who complained of hair loss during treatment with sertraline within only 2 months of treatment (at the beginning of treatment) while she was receiving a low dose of sertraline (unlike the other case).

Conclusions: SSRIs-induced hair loss is an infrequently observed adverse effect that may be overlooked. These case reports reinforce the need for maintaining a high index of suspicion regarding any incident of sudden hair loss occurring in persons taking even low doses of SSRIs like sertraline. Hair loss should also be questioned during the control sessions in addition to other side effects as it may influence patient's outer appearance and negatively affect his/her adherence to treatment.
\end{abstract}

Keywords: Alopecia, Male, Selective Serotonin Re-Uptake Inhibitors, Sertraline, Side Effect

\section{Introduction}

Selective serotonin reuptake inhibitors (SSRIs) are widely used in psychiatric medicine. SSRIs, first introduced in 1988 with fluoxetine, have an adverse event profile that is very favorable compared to other antidepressant drug classes (although this side effect has been improved in later-generation compounds). These drugs have significantly broadened the horizon in psychopharmacological treatment of anxiety and mood as well as obsessive compulsive disorders (1). The primary pharmacological characteristic of these drugs is strong, selective inhibition of serotonin reuptake, which is a significant factor in their efficacy for treatment of depression. The positive effect of serotonin reuptake inhibition in depression is far stronger than that actuated by noradrenaline reuptake, alpha 1 adrenergic, and muscarinic cholinergic and histamine receptors (2).

Hair loss is a side effect rarely observed during the use of psychotropic drugs. Expansion of hair loss on scalp may be either local or widespread. Psychotropic medications are deemed to affect telogen phase, consequently leading hair loss (3).

It is argued that most drug-induced hair loss is due to the conversion of growing hair follicles into resting hair follicles $(3,4)$. Shedding of the hair shaft takes place up to 3 months after the initiation of treatment (3).
Most people have about 110,000 hairs on scalp. Loss of 100 hairs per day is a normal event. The term alopecia encompasses all forms of hair loss ranging from simple effluvium to total loss of hair. Thinning of the hair is difficult to be detected by clinician until the loss of about $25 \%-50 \%$ of hairs on his/her scalp. Hair loss is, therefore, a subjective complaint of the person, who notices an increased hair loss while washing or brushing his/her hair (3). Medicines frequently cause hair loss as a side effect though this side effect is relatively rare with psychotropic agents (3). Association of SSRIs with hair loss is rare and limited to a few of case reports (5). All SSRIs have rarely been associated with hair loss (4). Clinicians often do not ask patients for this adverse effect and therefore may not detect its appearance. Conversely, patients recognize the side effect but generally cannot connect the hair loss to the SSRI they are using. The real prevalence of hair loss due to psychotropic medications is not obvious (6). As hair loss shows direct, bad influences on appearance, it can make an important problem for person; in cases where doctors are overlooking or missing this side effect, patient non-adherence to the SSRI regimen can be resulted. A few case reports exist on hair loss associated with tricyclic antidepressants and SSRIs used in combination (7). Fluoxetine (8-10), sertraline $(5,11,12)$, paroxetine (7) and serotonin-norepinephrine reuptake inhibitor (SNRI) venlafaxine (13) have been reported to cause hair loss in a small percent of patients. The mechanism of 
hair loss due to SSRIs has not been elucidated.

In this case study, a patient developed diffuse hair loss during sertraline treatment. This patient had not a family history and not a personal history of alopecia. Decreasing the dose of sertraline led to the resolution of hair loss within 6 weeks.

\section{Case Presentation}

Case I: Mr. A, a 47-year-old married man with a college diploma, was assessed during a psychiatric consultation after admission to an internal medical ward. During admission, the patient stated that he experienced dyspnea, with multiple attacks, and a general hospital admission for the condition over previous 4 months. Examination of the patient's mental state revealed good self-care with full orientation and cooperation; the patient was conscious; His mood was euthymic and the content of his thought corresponded with his mood. The patient received a diagnosis of panic disorder according to the DSM-V criteria and specific phobia (claustrophobia). He did not fulfill the DSM-V criteria for any other Axis I or Axis II. No prior history of suicide attempts or comorbid alcohol or substance abuse existed. In the absence of any accompanying Axis I pathological mental disorder (e.g., trichotillomania), other psychiatric illnesses that may cause hair loss were excluded. Physical examination was normal; the patient had a history of asthma (i.e., the already-noted dyspneic episodes). He had not been treated previously with any other SSRI. His sertraline regimen had been initiated at a dose of $25 \mathrm{mg} /$ day and gradually increased to $100 \mathrm{mg}$ daily. 5 weeks later, symptoms of panic attacks were dramatically reduced on this drug regimen along with the use of alprazolam. This treatment was associated with a marked response (i.e., significant change in the clinical global impressions-improvement scale (CGI-I)). Approximately 8 weeks after starting sertraline, he noticed hair loss as he brushed or washed his hair (scalp hair appearing in his comb and pillow). This observation was confirmed by the patient's hairdresser. His hair loss worsened progressively in the following weeks after this initial 8-week period. He had no itching or changes in the scalp; scalp pain did not accompany this non-cicatricial hair loss. He was not found to have non-cicatricial alopecia in patches around areas of scalp. Other than sertraline and alprazolam, the patient was taking salbutamol; he had no histories of allergies. He denied any changes in diet or in shampoos or soaps used, and was unaware of any drug allergies. In his familial and personal history, there was no history of hair loss at early ages. He denied using any herbal or homeopathic remedies or over-the-counter drug products. The patient denied tobacco, alcohol, or caffeine use.
After consultation with the dermatology clinic, the patient received a diagnosis of hair loss with unknown cause. He was referred to a dermatologist, but no other dermatological abnormality was found. The results of hematological assays and renal, liver function tests, thyroid function tests, erythrocyte sedimentation rate (ESR), and urinalysis revealed no abnormality. Thyroid antibodies and antinuclear antibodies (ANA) for lupus were not found. Serological tests confirmed that he did not have syphilis. Rheumatoid factors were not present. Considering that there was no pre-treatment period for hair loss and the exclusion of other potential causes such as positive family history and organic causes, sertraline use was considered to be a probable cause of the hair loss. With the suspicion of a drugrelated adverse event, the dose of sertraline was dropped to $75 \mathrm{mg} /$ day, and a zinc sulfate dose of $50 \mathrm{mg} /$ day initiated. Zinc sulfate was used because chelation of zinc may occur in some cases of hair loss induced by antipsychotics, leading to the depletion of zinc levels $(3,4)$. Zinc levels were not determined in this patient. Zinc sulfate has been advised in the treatment of alopecia, but the efficacy has not been established. Over a 4-week period, an increase in hair growth was reported by the patient in areas where hair growth had been affected. It is unknown whether zinc sulfate contributed to regrowth of hair or whether the regrowth resulted from decreasing the sertraline dose.

Four weeks after decreasing the dosage of sertraline, the patient noticed that progression of hair loss stopped; his hair re-grew within 2 months. After cessation of hair loss, the patient's hair returned back to its normal thickness with renewed hair growth.

Case II: Ms. M, a 32-year-old single woman, had been diagnosed with DSM-V Major depressive disorder with anxious distress. She did not fulfill DSM-V criteria for any other Axis I disorder Such as trichotillomania or Body dysmorphic disorder. She was medically healthy during the time of treatment for Major depressive disorder. The therapy with sertraline initiated for her with $25 \mathrm{mg} /$ day and then increased to $50 \mathrm{mg} /$ day within 2 months. Ms. A achieved partial remission of depressive symptoms. She noted hair loss within the first 2 months of treatment with sertraline $50 \mathrm{mg}$. The patient had not experienced hair loss before and she had no history of endocrine illness. A comprehensive work-up of chemistry profile, blood counts, thyroid function tests, liver functions tests, and ANA titers was normal. A dermatologist consultant reported no specific cause for her complains. She eventually became concerned enough to stop treatment completely. At this point, we decided to start the treatment of Ms. A with fluoxetine due to her inability to tolerate hair loss. Six weeks after stopping sertraline treatment and replacing by fluoxetine treatment, the hair loss ceased and her hair returned to 
normal thickness.

\section{Discussion}

Various clinical conditions can cause hair loss, including scalp diseases, systemic or infectious diseases, toxic agents, hormonal imbalances, and psychiatric disorders. Hair loss in the present patients is likely caused by sertraline, since hair loss appeared after increasing the dose of sertraline and improved after the dose reduction. Information about drug-induced alopecia is sparse and limited to case reports in medical literature. Drug-induced hair loss is more common than what is realized by many clinicians. Few details are available in this context, and hair loss secondary to drug use is often difficult to confirm (12).

The exact prevalence of hair loss with SSRI treatment has not been determined. In fact, quantifying this prevalence is likely not possible; it is difficult to diagnose hair loss due to drug use because no special method exists to arrive at this diagnosis. The only way to confirm the diagnosis is to stop the medication and observe hair regrowth (6). For the differential diagnosis, it is necessary to consider trichotillomania, hypothyroidism, hyperthyroidism, hormonal pathologies of the hypothalamic-pituitary-gonadal axis, and iron deficiency. It is also crucial to investigate the potential use by a given patient of other drugs related to hair loss (antihypertensive agents, anticoagulants, anticonvulsants, non-steroidal anti-inflammatory drugs, antiulcer agents) (14). For this reason, other conditions associated with hair loss must be rejected during differential diagnosis.

The pathological mechanism of hair loss due to psychotropic drugs has not yet been fully elucidated. The direct toxic effects of psychotropic drugs on the hair follicle matrix must be considered as a possible reason for hair loss in this case (15). The mechanism of drug-induced hair loss is termed telogen effluvium that involves a premature interruption of growth with early entry of anagen follicles into the resting phase. Hair loss tends to occur within the first 3 months of initiating therapy but may be further delayed $(3,4)$. It has also been shown that human skin can produce serotonin and transform it into melatonin, which has been implicated in hair-growth cycle. Therefore, it is possible that treatments interfering with serotonin homeostasis in the skin may alter the balance between hair growth and hair shedding (16). These data suggest a pathophysiological cause of hair loss with the use of antidepressant drugs that has not yet been fully identified (4). Given this, it is known that most psychotropic medication causes hair loss in the telogen phase of the hair cycle (4). Hair loss observed in patients receiving antidepressant drug treatment potentially relates to personal sensitivity rather than the drug administered (5). Although these drugs have an effect on zinc and selenium chelates, several obstacles has faced explaining this effect, namely the inefficiency of zinc and selenium supplements in treatment and the lack of information on the effect of cellular mechanism (17). Most drugs cause alopecia likely due to making the hair follicles to enter the resting phase. Therefore, decreasing the dose (or halting treatment completely) could potentially reverse this trend and lead to hair regrowth $(3,4)$.

A variety of serotonin reuptake inhibitors have been shown to cause hair loss, though this side effect is generally rare. The risk of alopecia seems to vary between different SSRIs (18), and SSRI-induced hair loss is associated with an individual, rather than a drug-specific, sensitivity (4). Sertraline can cause hair loss; sertraline and fluoxetine may have different effects on hair loss due to different dopaminergic effects; other SSRIs have been shown to rarely cause hair loss. Sertraline's relative potency for dopamine reuptake inhibition is one of its points of differentiation from other SSRIs such as fluoxetine (19). The different influence exerted by sertraline and fluoxetine on dopamine reuptake inhibition was identified as a possible reason for the side effect of hair loss. In fact, commonly prescribed prescription medications (such as dopaminergic agents) can cause temporary hair loss. In reviewing literature, there have been rarely reports of hair loss after administration of various antidepressant medications, including sertraline $(12,19)$.

A total of 27 reports of alopecia were identified in the SWEDIS drug database. As two reports concerned the use of two SSRIs, there were a total of 29 drug-ADR combinations, all but 3 of which were in women (88.9\%). The reported rate for alopecia in Sweden was significantly higher with sertraline compared with citalopram, i.e. 20.1 (95\% CI 10.7 - 34.4) reports per million patient-years versus 4.5 (95\% CI 1.8 - 9.3) reports per million patient-years (18).

Bourgeois published 2 cases of hair loss due to sertraline. The first case reportedly noticed hair loss approximately 6 weeks after starting sertraline $50 \mathrm{mg}$. In this case, when sertraline was replaced by paroxetine, hair loss stopped and the hair returned to normal thickness. The second case, a female patient who developed sertralinerelated alopecia (150 $\mathrm{mg} /$ day), discontinued the drug 2 months after noticing the side effect and switched the treatment to trazodone therapy with no recurrence of hair loss (11).

Ghanizadeh (19) reported a patient with a major depressive disorder who complained of diffuse scalp hair loss followed by treatment with sertraline. This case is unique because the patient had taken fluoxetine without reporting hair loss both before taking sertraline and after discontinuation. Uzun and colleagues (5) reported that hair 
loss developed in one patient following sertraline use and ceased within around 3 months after sertraline discontinuation. Turkoglu (12) reported that extensive hair loss developed with both sertraline and fluoxetine therapy in one case while it then ceased after the patient discontinued these two medications in favor of venlafaxine.

Clinical detection of hair loss is challenging until 25\% to $50 \%$ of a patient's hair is lost. Therefore, hair loss is a subjective complaint and it is mostly observed during washing or combing of the hair (3). Definitively concluding that a given drug causes hair loss presents specific challenges, as no special method exists for arriving at this diagnosis. Rather, discontinuing a drug (or reducing its dose) is often the only way to establish a causal connection to hair loss (20). In our case, hair re-grew after the sertraline dose decreased, indicating that hair loss was induced by sertraline. During differential diagnosis, the following should be considered and ruled out: trichotillomania; hypothalamicpituitary-gonadal axis hormone disorders; hypo/ hyperthyroidism; iron and copper deficiencies; menopause ; and use of oral contraceptives and other drugs that potentially cause hair loss, such as antihypertensive, anticoagulant, anticonvulsive, non-steroid anti-inflammatory, and antiulcer drugs (21). Necessary biochemical and endocrinological assays were also conducted on our patient, with the aforementioned considerations excluded along with renal and hepatic insufficiency and hepatitis in the process of differential diagnosis No dermatological illness that could cause hair loss was observed in our case. Observation of hair growth following the rule out of other differential diagnoses and lowering the medication led us to the conclusion that hair loss in our case related to sertraline. In terms of etiology, another interesting point in our case is that, while the majority of reported cases of antidepressantinduced hair loss are in women, our case study was a middle-aged man and it is therefore atypical in terms of gender; in Hedenmalm (18) study, all except 3 cases were in women. The previously published case reports on SSRIs and alopecia also predominantly reviewed cases in women $(7,9,11,22-25)$. It is possible that women may be more preoccupied with their hair and more prone to report hair loss to their physicians. Hair loss may also be more noticeable if the hair is long; some men already have a limited amount of hair. Despite these considerations, it is also plausible that women have an increased risk of SSRI-induced alopecia compared with men, even after correcting for the fact that more women than men are treated with SSRIs (19).

Drug-induced hair loss generally resolves (with recovery of hair growth) within 2 months after discontinuation of the drug (3). When an effective psychotherapeutic agent causes alopecia and no appropriate alternative can be provided, the informed patient and clinician should discuss the risks and benefits of continuing, stopping, or changing the dose or medication. Hair loss may be a rare side effect of SSRIs, but it is considered particularly traumatic for both men and women (6). Necessary measures must be taken to obviate this side effect when it emerges, including review of the advantages and disadvantages of maintaining treatment with the drug potentially causing the side effect. Significant hair loss should be monitored in treatment because it may lead to bad compliance non-adherence and relapse. Future clinical experience and more research may further clarify drug-induced hair loss and offer new therapeutic recommendations.

Alopecia induced by medications is generally characterized by a diffuse, non-scarring hair loss and its reversibility after stopping drug. Dosage reduction, drug stopping or pursuing therapy with another agent remains the most promising management option (26).

\subsection{Conclusion}

Hair loss related to SSRIs is an infrequently observed adverse effect that may be overlooked. We believe that hair loss should also be investigated during control visits in addition to other adverse effects as it may damage the patient's outer general appearance and negatively affect his or her adherence to management. The cause of this hair loss has not yet been fully elucidated; Researches on larger patient groups that aim to develop the potential mechanism of hair loss are required. Further investigation is needed to determine the scope of this troubling side effect.

\section{Footnotes}

Authors' Contribution: Forouzan Elyasi performed the psychiatric evaluation, collected and interpreted the clinical data, wrote the primary manuscript and revised it, and approved the final manuscript.

Funding/Support: None declared.

Declaration of Interest: None declared.

\section{References}

1. Kaplan HI, Sadock BJ. Kaplan \& Sadock' s Synopsis of Psychiatry. Biological Therapies. Baltimore: Lippincott Williams \& Wilkins; 2015. pp. 1083-92.

2. Croker CG, Pearcy JO, Stahl DC, Moore RE, Keen DA, Lee TD. An expert virtual instrument approach to the automated, data dependent MS/MS and LC/MS/MS analysis of proteins. J Biomol Tech. 2000;11(3):135-41. [PubMed:19499049].

3. Gautam M. Alopecia due to psychotropic medications. Ann Pharmacother. 1999;33(5):631-7. [PubMed: 10369629].

4. Mercke Y, Sheng H, Khan T, Lippmann S. Hair loss in psychopharmacology. Ann Clin Psychiatry. 2000;12(1):35-42. [PubMed: 10798824].

5. Uzun O, Cansever A, Ozgen F. Hair loss due to sertraline use: a case report. Bulletin of Clinical Psychopharmacol. 2003;13:27-9. 
6. Warnock JK. Psychotropic medication and drug-related alopecia. Psychosomatics. 1991;32(2):149-52. doi: 10.1016/S0033-3182(91)72085-9. [PubMed: 2027936].

7. Zalsman G, Sever J, Munitz H. Hair loss associated with paroxetine treatment: a case report. Clin Neuropharmacol. 1999;22(4):246-7. [PubMed: 10442258].

8. Jenike MA. Severe hair loss associated with fluoxetine use. Am JPsychiatry. 1991;148(3):392. [PubMed: 1992847].

9. Ogilvie AD. Hair loss during fluoxetine treatment. Lancet. 1993;342(8884):1423. [PubMed: 7901703].

10. Mermi O, Atmaca M, Kilic FM, Gurok G, Kuloglu M. Fluoxetine Induced Hair Loss: A Case Report. Dusunen Adam. J Psychiatr Neurol Sci. 2014;27(3).

11. Bourgeois JA. Two cases of hair loss after sertraline use. J Clin Psychopharmacol. 1996;16(1):91-2. [PubMed: 8834433].

12. Turkoglu S. Fluoxetine and sertraline related hair loss in a teenager: a case report. Bulletin Clin Psychopharmacol. 2013;23:77-80. doi: 10.5455/bcp.20120928030857.

13. Pitchot W, Ansseau M. Venlafaxine-induced hair loss. Am J Psychiatry. 2001;158(7):1159-60. [PubMed: 11431245].

14. McKinney PA, Finkenbine RD, DeVane CL. Alopecia and mood stabilizer therapy. Ann Clin Psychiatry. 1996;8(3):183-5. [PubMed: 8899137].

15. Piraccini BM, Iorizzo M, Rech G, Tosti A. Drug-induced hair disorders. Curr Drug Saf. 2006;1(3):301-5. [PubMed: 18690941].

16. Slominski A, Wortsman J, Tobin DJ. The cutaneous serotoninergic/melatoninergic system: securing a place under the sun. FASEB J. 2005;19(2):176-94. doi: 10.1096/fj.04-2079rev. [PubMed: 15677341].
17. Leung M, Wrixon $\mathrm{K}$, Remick RA. Olanzapine-induced hair loss. Can J Psychiatry. 2002;47(9):891-2. [PubMed: 12500769].

18. Hedenmalm K, Sundstrom A, Spigset O. Alopecia associated with treatment with selective serotonin reuptake inhibitors (SSRIs). Pharmacoepidemiol Drug Saf. 2006;15(10):719-25. doi: 10.1002/pds.1270. [PubMed: 16783834].

19. Ghanizadeh A. Sertraline-associated hair loss. J Drugs Dermatol. 2008;7(7):693-4. [PubMed: 18664165].

20. Pereira CE, Goldman-Levine JD. Extended-release venlafaxineinduced alopecia. Ann Pharmacother. 2007;41(6):1084. doi: 10.1345/aph.1G442. [PubMed: 17519298].

21. Yucel A, Karakus G, Gunasti S. Dermatological side effects of psychotropic drugs and treatment approaches. Bulletin Clin Psychopharmacol. 2008;18:235-44.

22. Bhatara VS, Gupta S, Freeman JW. Fluoxetine associated paresthesias and alopecia in a woman who tolerated sertraline. J Clin Psychiatry. 1996;57(5):227. [PubMed: 8626355].

23. Seifritz E, Hatzinger M, Muller MJ, Hemmeter U, Holsboer-Trachsler E. Hair loss associated with fluoxetine but not with citalopram. Can J Psychiatry. 1995;40(6):362. [PubMed: 7585411].

24. Gupta S, Major LF. Hair loss associated with fluoxetine. Br J Psychiatry. 1991;159:737-8. [PubMed: 1756373].

25. Mareth TR. Hair loss associated with fluoxetine use in two family members. J Clin Psychiatry. 1994;55(4):163. [PubMed: 8071263].

26. Ceylan MF, Yalcin O. Atomoxetine-related hair loss in a teenager: a case report. Bulletin Clin Psychopharmacol. 2010;20(3):258-60. 